\title{
Unusual high blood glucose in ketoacidosis as first presentation of type 1 diabetes mellitus
}

\author{
Sebastian Hörber1,2,3, Sarah Hudak1, Martin Kächele4, Dietrich Overkamp', \\ Andreas Fritsche1,2,3, Hans-Ulrich Häring1,2,3, Andreas Peter1,2,3 and Martin Heni1,2,3 \\ 1Division of Endocrinology, Diabetology, Vascular Medicine, Nephrology and Clinical Chemistry, Department of \\ Internal Medicine, University of Tübingen, Tübingen, Germany, 2Institute for Diabetes Research and Metabolic \\ Diseases of the Helmholtz Center Munich at the University of Tübingen, Tübingen, Germany, ${ }^{3 G}$ German Center for \\ Diabetes Research (DZD), München-Neuherberg, Germany, and ${ }^{4}$ Department of Internal Medicine, Medical Intensive \\ Care Unit, University of Tübingen, Tübingen, Germany
}

\author{
Correspondence \\ should be addressed \\ to M Heni \\ Email \\ Martin.Heni@med. \\ uni-tuebingen.de
}

\section{Summary}

Diabetic ketoacidosis is a life-threatening complication of diabetes mellitus. It usually occurs in patients with type 1 diabetes where it is typically associated with only moderately increased blood glucose. Here, we report the case of a 52-year-old female patient who was admitted to the emergency unit with severely altered mental status but stable vital signs. Laboratory results on admission revealed very high blood glucose $(1687 \mathrm{mg} / \mathrm{dL} / 93.6 \mathrm{mmol} / \mathrm{L})$ and severe acidosis $(\mathrm{pH}<7)$ with proof of ketone bodies in serum and urine. Past history revealed a paranoid schizophrenia diagnosed 10 years ago and for which the patient was treated with risperidone for many years. Acute treatment with intravenous fluids, intravenous insulin infusion and sodium bicarbonate improved the symptoms. Further laboratory investigations confirmed diagnosis of autoimmune type 1 diabetes. After normalization of blood glucose levels, the patient could soon be discharged with a subcutaneous insulin therapy.

\section{Learning points:}

- Diabetic ketoacidosis as first manifestation of type 1 diabetes can occur with markedly elevated blood glucose concentrations in elder patients.

- Atypical antipsychotics are associated with hyperglycemia and an increased risk of new-onset diabetes.

- First report of risperidone-associated diabetic ketoacidosis in new-onset type 1 diabetes.

- Patients treated with atypical antipsychotics require special care and regular laboratory examinations to detect hyperglycemia and diabetic ketoacidosis.

- In cases when the diagnosis is in doubt, blood gas analysis as well as determination of C-peptide and islet autoantibodies can help to establish the definite diabetes type.

\section{Background}

High blood glucose levels $(>600 \mathrm{mg} / \mathrm{dL} / 33.3 \mathrm{mmol} / \mathrm{L}$ ) are a typical finding in hyperglycemic hyperosmolar syndrome, mostly seen in elderly patients with longstanding type 2 diabetes. It is characterized by high blood glucose, increased serum osmolarity and dehydration without significant ketoacidosis and therefore normal
pH (1). In contrast, diabetic ketoacidosis (DKA) usually occurs in younger patients with type 1 diabetes and is associated with only moderate increased blood glucose (250-600 mg/dL/13.9-33.3 mmol/L) (2). DKA is defined by metabolic acidosis, high blood glucose, and the presence of ketone bodies in blood and urine. Reduced 
insulin effect due to markedly reduced or absent insulin secretion is the major cause for hyperglycemia in this condition. The inability of glucose to enter the cells leads to increased stimulation of lipolysis and subsequent accumulation of free fatty acids and significant amounts of ketone bodies (3). As a consequence, counter regulatory hormones, such as catecholamines, glucagon and cortisol are upregulated and act in direct opposition to insulin (4). Normally, elderly patients with very high blood glucose levels are considered to have type 2 diabetes and the high prevalence of type 2 diabetes in patients at that age makes it challenging to identify type 1 diabetes patients among them (5).

Risperidone is an atypical antipsychotic approved for the treatment of schizophrenia. It is long known that atypical antipsychotics are linked to hyperglycemia, new-onset of type 2 diabetes and in rare cases also with the development of diabetic ketoacidosis (6). Patients on atypical antipsychotic medication should therefore be considered as patients at high risk for diabetes and presumably also DKA.

\section{Case presentation}

A 52-year-old female patient was admitted to the emergency unit with severely altered mental status. The patient's son reported that the patient complained of abdominal pain, nausea and vomiting for 2 days. The day before admission, the patient introduced herself to an ambulatory emergency service due to constant restlessness. They prescribed diazepam when needed. On admission to the emergency unit, the patient was somnolent. Vital signs revealed mild tachycardia (112/ min), normal blood pressure $(115 / 75 \mathrm{mmHg})$ and hyperventilation (Kussmaul breathing). Body temperature was normal. The patient was lean with a BMI of $21.7 \mathrm{~kg} /$ $\mathrm{m}^{2}$. Past history revealed no preexisting conditions, except for a paranoid schizophrenia diagnosed 10 years ago. Therefore, she has been treated with risperidone for many years. Family history was negative for diabetes and other relevant diseases.

\section{Investigations}

Initial laboratory results included (Fig. 1 and Table 1): a reduced blood $\mathrm{pH}$ of $<7$, a high serum anion gap (41 mEq/dL), markedly elevated blood glucose $(1687 \mathrm{mg} /$ $\mathrm{dL} / 93.6 \mathrm{mmol} / \mathrm{L}$ ), elevated lactate of $5.1 \mathrm{mg} / \mathrm{dL}$ and serum osmolality of $404 \mathrm{mosmol} / \mathrm{kg}$. The urine sample was clearly positive for ketones and glucose. A drug screening
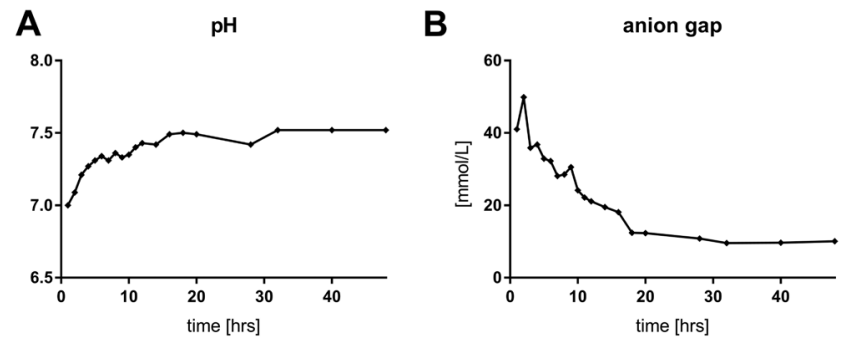

Figure 1

Arterial blood gas analysis. (A) Time course of the $\mathrm{pH}$ measurements for the first 2 days after admission. (B) Time course of the serum anion gap. Shown are values in $\mathrm{mmol} / \mathrm{L}$ for the first 2 days after admission. The anion gap is calculated by subtracting the serum concentrations of chloride and bicarbonate from the concentrations of sodium and potassium.

was negative except for benzodiazepines. Serum betahydroxybutyrate was elevated and $\mathrm{HbA1c}$ was $12.2 \%$ $(110 \mathrm{mmol} / \mathrm{mol})$. C-peptide was decreased $(77 \mathrm{pmol} / \mathrm{L})$.

\section{Treatment}

Due to positive proof of benzodiazepine in urine and severely altered mental status, the treatment was first started with the benzodiazepine-antagonist flumazenil whereupon her condition improved only slightly. Though, she was simultaneously treated with intravenous fluids, intravenous insulin infusion and sodium bicarbonate under constant control of vital parameters, blood glucose, electrolytes and blood gas analyses.

\section{Outcome and follow-up}

Over the next $48 \mathrm{~h}$, her general condition dramatically improved, blood glucose level fell and the anion gap normalized (Fig. 1 and Table 1). Immunological investigations revealed pancreatic autoantibodies against glutamic acid decarboxylase (GAD). In line with the markedly reduced C-peptide concentrations, we diagnosed type 1 diabetes. After normalization of blood glucose levels, the patient was transferred from the intensive care unit to the general ward and could soon be discharged from the hospital with a subcutaneous insulin injection therapy.

\section{Discussion}

Our patient clearly suffered from DKA due to newly diagnosed type 1 diabetes. Previously, auto-antibodypositive diabetes was sometimes also classified as latent autoimmune diabetes in the adult (LADA). Though, 
Table 1 Time course of arterial blood gas analyses and basic laboratory results.

\begin{tabular}{|c|c|c|c|c|c|c|c|c|c|c|}
\hline & Reference range & Admission & $1 \mathrm{~h}$ & $2 \mathbf{h}$ & $4 h$ & $8 h$ & $16 \mathrm{~h}$ & $24 h$ & Day 3 & Day 5 \\
\hline \multicolumn{11}{|l|}{ Blood gas analyses } \\
\hline $\mathrm{pH}$ & $7.35-7.45$ & $<7.0$ & 7.09 & 7.21 & 7.31 & 7.36 & 7.40 & 7.47 & & \\
\hline Sodium (mmol/L) & 136-148 & 125 & 130 & 144 & 146 & 146 & 150 & 151 & & \\
\hline Potassium (mmol/L) & $3.5-4.8$ & 6.9 & 5.7 & 6.7 & 4.2 & 4.9 & 3.5 & 4.1 & & \\
\hline Chloride (mmol/L) & $96-110$ & 78 & 80 & 107 & 109 & 111 & 115 & 116 & & \\
\hline Bicarbonate (mmol/L) & $22.0-26.0$ & 4.8 & 5.8 & 7.8 & 8.3 & 11.4 & 16.3 & 24.1 & & \\
\hline $\mathrm{pO}_{2}(\mathrm{mmHg})$ & 65-100 & 94 & 125 & 122 & 129 & 112 & 109 & 92 & & \\
\hline $\mathrm{pCO}_{2}(\mathrm{mmHg})$ & $32.0-42.0$ & 20 & 19.9 & 44 & 13.6 & 25.8 & 27.0 & 34.0 & & \\
\hline Anion gap (mEq/L) & 3-11 & 49.1 & 49.9 & 35.9 & 32.9 & 28.5 & 22.2 & 15.0 & & \\
\hline Base excess (mmol/L) & -3.0 to 3.0 & -23.2 & -21.5 & -11.7 & -19.3 & -14.2 & -7.5 & 0.1 & & \\
\hline Glucose (mg/dL) & 70-90 & 1687 & 1232 & 1059 & 890 & 837 & 845 & 806 & & \\
\hline \multicolumn{11}{|l|}{ Laboratory tests } \\
\hline Hematocrit (\%) & $37.0-47.0$ & 43.3 & & & & & & & 26.1 & 36.8 \\
\hline Hemoglobin (g/dL) & $12.0-16.0$ & 13.2 & & & & & & & 9.7 & 12.8 \\
\hline White cell count (per mm³) & $4100-11800$ & 16740 & & & & & & & 8700 & 5550 \\
\hline Platelet count $\left(10^{3}\right.$ per $\left.\mathrm{mm}^{3}\right)$ & $150-450$ & 319 & & & & & & & 137 & 218 \\
\hline PT (INR) & & 0.9 & & & & & & & 1.1 & 1.0 \\
\hline aPTT (s) & Max. 40 & 27 & & & & & & & & \\
\hline \multicolumn{11}{|l|}{ Electrolytes (mmol/L) } \\
\hline Sodium & $136-148$ & 124 & & & & & & & 148 & 140 \\
\hline Potassium & $3.5-4.8$ & 6.5 & & & & & & & 4.1 & 4.1 \\
\hline Calcium & $2.1-2.6$ & 2.4 & & & & & & & 2.0 & \\
\hline Phosphate & $0.8-1.5$ & 4.2 & & & & & & & 0.8 & 1.3 \\
\hline Chloride & $96-110$ & 75 & & & & & & & 113 & 109 \\
\hline Creatinine (mg/dL) & $0.5-0.8$ & 2.9 & & & & & & & 0.6 & 0.6 \\
\hline GFR MDRD $\left(\mathrm{mL} / \mathrm{min} * 1.73 \mathrm{~m}^{2}\right)$ & $80 \pm 24$ & 17 & & & & & & & 105.0 & 105.0 \\
\hline C-reactive protein (mg/dL) & Max. 0.5 & 0.4 & & & & & & & 1.4 & 0.34 \\
\hline Creatin kinase (mg/dL) & Max. 170 & 43 & & & & & & & 239 & 161 \\
\hline Urea (mg/dL) & $12-46$ & 108 & & & & & & & 8 & \\
\hline Total cholesterol (mg/dL) & $130-190$ & & & & & & & & & 239 \\
\hline Triglycerides (mg/dL) & $<200$ & & & & & & & & & 111 \\
\hline LDL cholesterol (mg/dL) & $<160$ & & & & & & & & & 175 \\
\hline HDL cholesterol (mg/dL) & $>45$ & & & & & & & & & 51 \\
\hline Total protein (g/dL) & $6.5-8.5$ & 4.3 & & & & & & & & 7.2 \\
\hline Albumin (g/dL) & $3.0-5.0$ & 2.9 & & & & & & & & \\
\hline Alanine transaminase (U/L) & Max. 34 & 15 & & & & & & & & \\
\hline Alkaline phosphatase (U/L) & $35-105$ & 90 & & & & & & & & \\
\hline Gamma-glutamyl transferase (U/L) & Max. 40 & 14 & & & & & & & 9 & \\
\hline Lipase (U/L) & Max. 60 & 80 & & & & & & & 40 & \\
\hline Lactate dehydrogenase (U/L) & Max. 250 & 133 & & & & & & & 162 & \\
\hline Serum osmolality (mosmol/kg) & $275-300$ & 404 & & & & & & & 321 & \\
\hline TSH (mU/L) & $0.5-4.4$ & 0.93 & & & & & & & & \\
\hline Cortisol (nmol/L) & $130-630$ & 3446 & & & & & & & 429 & \\
\hline Lactate $(\mathrm{mg} / \mathrm{dL})$ & $0.5-2.2$ & 5.1 & & & & & & & 1.6 & \\
\hline C-peptide (pmol/L) & $140-830$ & 77 & & & & & & & & 114 \\
\hline Glucose (mg/dL) & 70-99 & 1687 & & & & & & & & 331 \\
\hline $\begin{array}{l}\text { Glycosylated hemoglobin (\%/IFCC) } \\
\text { (mmol/mol) }\end{array}$ & $4.5-6.2 / 26-44$ & $12.2 / 110$ & & & & & & & & \\
\hline GAD antibodies (IU/mL) & $<5$ & 135.0 & & & & & & & & \\
\hline IA2 antibodies (IU/mL) & $<10$ & $<0.8$ & & & & & & & & \\
\hline 3-Hydroxy butyric acid (mg/dL) & $3-9$ & 1149 & & & & & & & & 9 \\
\hline Acetoacetic acid (mg/dL) & $<20$ & 495 & & & & & & & & 2 \\
\hline
\end{tabular}

Numbers in bold were outside the reference range.

aPTT, activated partial thromboplastin time; BE, base excess; GAD, glutamic acid decarboxylase; GFR, glomerular filtration rate; HDL, high-density lipoprotein; IA, islet antigen; IFCC, international federation of clinical chemistry; INR, international normalized ratio; LDL, low-density lipoprotein; MDRD, modification of diet in renal disease; $\mathrm{pCO}_{2}$, partial pressure of $\mathrm{CO}_{2} ; \mathrm{PO}_{2}$, partial pressure of $\mathrm{O}_{2}$; $\mathrm{PT}$, prothrombin time; TSH, thyroid-stimulating hormone; U, units. 
C-peptide concentrations were unusually low for this diabetes subtype (7), and the latest guidelines recommend classifying all cases of autoimmune diabetes as type 1 diabetes (8). Of notice, there is a lot of discussion in the field of diabetes subclassification lately, due to novel computational approaches on this topic (9). To our knowledge, our report is the first report about risperidoneassociated DKA in new-onset type 1 diabetes. Several reviews addressed this rare and potentially detrimental side effect of atypical antipsychotics. Though, almost all previous reports are related to dysregulated glucose homeostasis in patients with type 2 diabetes $(6,10)$. A few studies focused on the mechanism of action to understand the pathogenesis of atypical antipsychoticinduced diabetes. Weight gain, as a typical side effect of this drug class, and insulin resistance is considered to be a main factor that drives the association of atypical antipsychotics with hyperglycemia (11). However, our patient was lean and weight gain alone can therefore not fully explain this association. There are some reports about other antipsychotics, like risperidone that was prescribed to our patient or aripiprazole, which have less effect on body weight (12). Of notice, these substances have also been linked to diabetes risk. Recently, one report even described a new diagnosed type 1 diabetes subject treated with olanzapine (13). The authors hypothesized that olanzapine led to a relevant hyperglycemia, thereby accelerating the onset type 1 diabetes. The precise underlying mechanisms, however, remain elusive.

The development of blood glucose concentrations, as high as in our patient, is very unusual in the context of DKA. It is possible that the blood glucose levels had increased to that enormous extent because the symptoms might have been masked by the diazepam that was prescribed the day before admission. Nevertheless, the development and the precise mechanism of such high glucose values with delayed clinical symptoms also remain unclear.

\section{Conclusion}

Very high blood glucose levels are often indicative for type 2 diabetes. However, in rare cases, the first manifestation of type 1 diabetes can also present with markedly elevated glucose $(>1000 \mathrm{mg} / \mathrm{dL} / 55.5 \mathrm{mmol} / \mathrm{L})$. Failure to diagnose type 1 diabetes, especially in elderly patients, can have serious consequences due to initiation of inadequate treatment. In cases when the diagnosis is in doubt, blood gas analysis as well as determination of C-peptide and islet autoantibodies can help to establish the definite diabetes type. Furthermore, patients who are treated with atypical antipsychotics require special care and regular laboratory examinations to detect hyperglycemia and diabetic ketoacidosis.

\section{Declaration of interest}

The authors declare that there is no conflict of interest that could be perceived as prejudicing the impartiality of the research reported.

\section{Funding}

The authors acknowledge support by the Deutsche Forschungsgemeinschaft and the Open Access Publishing Fund of the University of Tübingen. Part of this work was supported by a grant (01GI0925) from the Federal Ministry of Education and Research (BMBF) to the German Centre for Diabetes Research (DZD e.V.).

\section{Author contribution statement}

Se $\mathrm{H}$, Sa H, M K, D O and M H looked after the patient and wrote the report. A F, A P and $\mathrm{H}-\mathrm{U} \mathrm{H}$ reviewed the report. All authors approved the final version before submission.

\section{References}

1 Pasquel FJ \& Umpierrez GE. Hyperosmolar hyperglycemic state: a historic review of the clinical presentation, diagnosis, and treatment. Diabetes Care 201437 3124-3131. (https://doi.org/10.2337/dc140984)

2 Kitabchi AE, Umpierrez GE, Miles JM \& Fisher JN. Hyperglycemic crises in adult patients with diabetes. Diabetes Care 200932 1335-1343. (https://doi.org/10.2337/dc09-9032)

3 Lebovitz HE. Diabetic ketoacidosis. Lancet 1995345 767-772. (https://doi.org/10.1016/S0140-6736(95)90645-2)

4 Kitabchi AE, Umpierrez GE, Murphy MB \& Kreisberg RA. Hyperglycemic crises in adult patients with diabetes: a consensus statement from the American Diabetes Association. Diabetes Care 200629 2739-2748. (https://doi.org/10.2337/dc06-9916)

5 Thomas NJ, Jones SE, Weedon MN, Shields BM, Oram RA \& Hattersley AT. Frequency and phenotype of type 1 diabetes in the first six decades of life: a cross-sectional, genetically stratified survival analysis from UK biobank. Lancet Diabetes Endocrinology 20176 122-129. (https://doi.org/10.1016/S2213-8587(17)30362-5)

6 Guenette MD, Hahn M, Cohn TA, Teo C \& Remington GJ. Atypical antipsychotics and diabetic ketoacidosis: a review. Psychopharmacology 2013226 1-12. (https://doi.org/10.1007/s00213013-2982-3)

7 Ahlqvist E, Storm P, Karajamaki A, Martinell M, Dorkhan M, Carlsson A, Vikman P, Prasad RB, Aly DM, Almgren P, et al. Novel subgroups of adult-onset diabetes and their association with outcomes: a data-driven cluster analysis of six variables. Lancet Diabetes Endocrinology 20186 361-369. (https://doi.org/10.1016/ S2213-8587(18)30051-2)

8 American Diabetes Association. 2. Classification and diagnosis of diabetes: standards of medical care in Diabetes-2018. Diabetes Care 201841 S13-S27. (https://doi.org/10.2337/dc18-S002)

9 Sladek R. The many faces of diabetes: addressing heterogeneity of a complex disease. Lancet Diabetes Endocrinology 20186 348-349. (https://doi.org/10.1016/S2213-8587(18)30070-6) 
10 Lipscombe LL, Austin PC, Alessi-Severini S, Blackburn DF, Blais L, Bresee L, Filion KB, Kawasumi Y, Kurdyak P, Platt RW, et al. Atypical antipsychotics and hyperglycemic emergencies: multicentre, retrospective cohort study of administrative data. Schizophrenia Research 2014154 54-60. (https://doi.org/10.1016/j.schres.2014.01.043).

11 Alvarez-Jimenez M, Gonzalez-Blanch C, Vazquez-Barquero JL, PerezIglesias R, Martinez-Garcia O, Perez-Pardal T, Ramirez-Bonilla ML \& Crespo-Facorro B. Attenuation of antipsychotic-induced weight gain with early behavioral intervention in drug-naive first-episode psychosis patients: a randomized controlled trial. Journal of Clinical
Psychiatry 200667 1253-1260. (https://doi.org/10.4088/JCP. v67n0812)

12 Baptista T, ElFakih Y, Uzcategui E, Sandia I, Talamo E, Araujo de Baptista E \& Beaulieu S. Pharmacological management of atypical antipsychotic-induced weight gain. CNS Drugs 200822 477-495. (https://doi.org/10.2165/00023210-200822060-00003)

13 Iwaku K, Otuka F \& Taniyama M. Acute-onset Type 1 diabetes that developed during the administration of olanzapine. Internal Medicine 201756 335-339. (https://doi.org/10.2169/ internalmedicine.56.7010)

Received in final form 6 August 2018

Accepted 20 August 2018 